\title{
Experimental Study on the Localization of Moving Object by Total Geomagnetic Field
}

\author{
Chong Kang, ${ }^{1}$ Liming Fan, ${ }^{2}$ Quan Zheng, ${ }^{1}$ Xiyuan Kang, ${ }^{3}$ Jian Zhou, ${ }^{2}$ and Xiaojun Zhang \\ ${ }^{1}$ College of Science, Harbin Engineering University, Harbin 150001, China \\ ${ }^{2}$ College of Mechanical and Electrical Engineering, Harbin Engineering University, Harbin 150001, China \\ ${ }^{3}$ College of Computer Science and Technology, Harbin Engineering University, Harbin 150001, China \\ Correspondence should be addressed to Chong Kang; kangchongheu@163.com
}

Received 10 March 2017; Revised 15 May 2017; Accepted 4 June 2017; Published 4 July 2017

Academic Editor: Eduard Llobet

Copyright (C) 2017 Chong Kang et al. This is an open access article distributed under the Creative Commons Attribution License, which permits unrestricted use, distribution, and reproduction in any medium, provided the original work is properly cited.

\begin{abstract}
In the method of target localization based on magnetic anomalies, the scheme of vector field localization and experimental research are significant. Because more information of magnetic field can be measured by vector sensors, the position of the target can be directly calculated by the equations. However, the vector magnetic anomaly generated by the target is difficult to measure. And the detection range is small due to the low sensitivity of vector sensors. A method for target localization based on the total geomagnetic field is proposed. Its advantages are that the measurement of total magnetic field is not affected by the orientation of the total field sensors and the detection range is large due to their high sensitivity. In this paper, we focus on the localization using the array with the total field magnetometers. And we design an array structure with the total field magnetometers. Then, we obtain the higher order nonlinear equations for the target localization based on this array. The numerical method is used to solve the equations. Meanwhile, we present a method for eliminating the effect of geomagnetic field variations and uneven spatial distribution. In suburban roads, localization experiments were carried out. And the results showed that the relative error of target localization is less than $5 \%$ by using the proposed method.
\end{abstract}

\section{Introduction}

Geomagnetic field is an important physical quantity, which reflects the evolution of the earth and the evolution of the geological structure. Due to the effect of the geomagnetic field, a target containing ferromagnetic material can generate a magnetic anomaly. It has an important application to locate the target based on the magnetic anomaly, which can be used in terms of energy mineral exploration, geological monitoring, shipwreck search and rescue, antisubmarine mines, medical diagnosis, and so forth. Therefore, the detection technology using magnetic anomalies generated by a target has become an important trend in target detection [1-7]. Usually, we can describe the target with six parameters, three describing the position and three describing the magnetic moments of the target. And the parameters of the target can be estimated by measuring the total magnetic field or the vector magnetic field.
The vector sensor can be used to measure the magnetic field vector. At present, the main vector magnetic sensors include the fluxgate magnetometer and SQUID magnetometer. In the research of target localization based on the magnetic anomaly, the use of a magnetic field vector is significant. Because the geomagnetic field is a vector field, vector measurement can be a comprehensive description of the geomagnetic element. And it has an advantage in the latter algorithm design. Nara et al. designed a magnetic sensor array for detecting the position of the object using the magnetic field and spatial gradients [3]. Marschner and Fischer located a magnetic object using a hall sensor array [8]. Liu and Wang proposed a method based on a 3-axis fluxgate sensor array for the localization of unexploded ordnances [9]. A vector sensor array must be used in all of these scenarios. When the vector sensor array is installed, the three axes of the sensors need to be strictly aligned, and the layout is complex. In addition, the orientation of the vector sensor can affect the measurement 


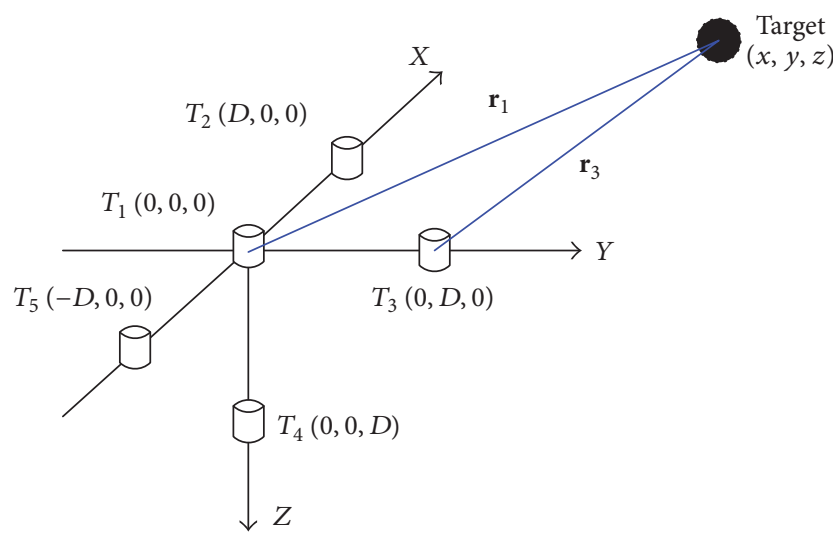

Figure 1: Schematic diagram of the array structure for magnetic target localization.

[10]. For example, if the geomagnetic field has magnitude of $55,000 \mathrm{nT}$ and the geomagnetic inclination is 60 degrees, the change of 0.05 degrees of pitch angle can lead to about $50 \mathrm{nT}$ component error of the geomagnetic field. Therefore, it is very difficult to locate the magnetic target using the sensor array with vector sensors when the array is installed on a moving vehicle. Meanwhile, the sensitivity of the vector sensor is low [11], probably in the nT level, and temperature will affect the measurement value. These reasons increase the difficulty of the localization of the remote target based on magnetic vector sensors.

Total field magnetometers have the important advantage of insensitivity to their orientation [11]. The optical pumped magnetometer is one kind of them, which is based on the Zeeman Effect. Since the temperature does not affect the atomic energy level, there is almost no temperature drift problem in the optical pumped magnetometer. In addition, the optical pumped magnetometer has high sensitivity at about fT level, which is especially important for locating small targets or remote targets. Therefore, it is useful to locate the remote magnetic target using an array with optical pumped magnetometers. In this study, we focused on the localization of magnetic targets using the array with the total field magnetometers. And we proposed a method for localization based on the total geomagnetic field and carried out experiments to verify the effectiveness of the proposed method. A method of eliminating the effect of geomagnetic field variations and uneven spatial distribution was also proposed.

\section{Principle of the Target Localization Based on the Total Geomagnetic Field}

2.1. Array and Algorithm for the Target Localization. When the distance between the target and the sensor is more than 2-3 times its own scale, the magnetic target can be considered as a magnetic dipole [12]. To estimate the position of the target and measure enough geomagnetic field, we designed an array with five total field sensors. The distance between the two adjacent sensors is $D$. The array with total field sensors is shown in Figure 1.
The magnetic moment of the magnetic target is synthesized by hard and soft magnetic moment [13]. The direction of the soft magnetic moment is parallel to the direction of the ambient magnetic field. And the direction of the hard magnetic moment is determined by the hard magnetic materials of the target. Normally, the soft magnetic moment is much larger than the hard magnetic moment. In this study, we only consider the soft magnetic moment of the target. In practice, the magnetic field $\mathbf{B}$ generated by the target is superimposed on the geomagnetic field. And the value $\mathbf{T}$ measured by the sensor contains the magnetic field and the geomagnetic field, shown in Figure 2. We transform the distance $r$ from the spherical coordinate system to the Cartesian coordinate system and obtain $x=r \cos \phi \sin \vartheta$, $y=r \sin \phi \sin \vartheta$, and $z=r \cos \vartheta$. According to them, the scalar expression of $\mathbf{T}$ is given as [14]

$$
\begin{aligned}
T_{1} & =T_{0}+\frac{\mu_{0}\left|\mathbf{P}_{m}\right|}{4 \pi r_{1}^{3}}[3 \\
& \left.\cdot \frac{(x \cos \varphi \cos \theta+y \sin \varphi \cos \theta+z \sin \theta)^{2}}{r_{1}^{2}}-1\right] \\
T_{2} & =T_{0}+\frac{\mu_{0}\left|\mathbf{P}_{m}\right|}{4 \pi r_{2}^{3}}[3 \\
& \cdot \frac{((x-D) \cos \varphi \cos \theta+y \sin \varphi \cos \theta+z \sin \theta)^{2}}{r_{2}^{2}} \\
& -1]
\end{aligned}
$$

$$
T_{3}=T_{0}+\frac{\mu_{0}\left|\mathbf{P}_{m}\right|}{4 \pi r_{3}^{3}}[3
$$$$
\cdot \frac{(x \cos \varphi \cos \theta+(y-D) \sin \varphi \cos \theta+z \sin \theta)^{2}}{r_{3}^{2}}
$$$$
-1]
$$

$$
\begin{aligned}
T_{4} & =T_{0}+\frac{\mu_{0}\left|\mathbf{P}_{m}\right|}{4 \pi r_{4}^{3}}[3 \\
& \cdot \frac{(x \cos \varphi \cos \theta+y \sin \varphi \cos \theta+(z-D) \sin \theta)^{2}}{r_{4}^{2}} \\
& -1] \\
T_{5} & =T_{0}+\frac{\mu_{0}\left|\mathbf{P}_{m}\right|}{4 \pi r_{5}^{3}}[3 \\
& . \frac{((x+D) \cos \varphi \cos \theta+y \sin \varphi \cos \theta+z \sin \theta)^{2}}{r_{5}^{2}} \\
& -1],
\end{aligned}
$$




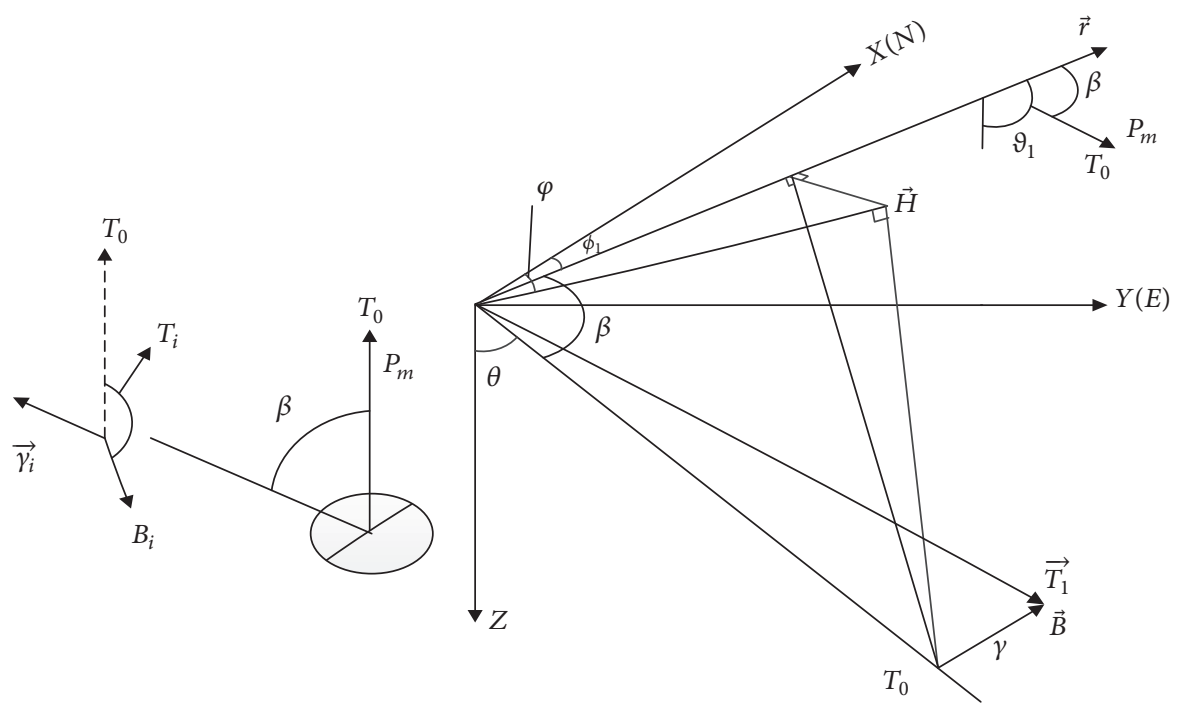

Figure 2: Principle diagram of the physical model of the geomagnetic field and magnetic moment.

where $\mu_{0}$ is the permeability in the free space. $\left|\mathbf{P}_{m}\right|$ denotes the magnitude of the magnetic moment. $(x, y, z)$ denotes the position of the target. $r_{i}$ denotes the distance between the target and the $i$ th sensor. $\theta$ and $\varphi$ denote the geomagnetic inclination and declination, respectively. $D$ is the distance between the two adjacent sensors.

When $T_{i}$ is measured by the $i$ th sensor, the values of target $\left(x, y, z,\left|\mathbf{P}_{m}\right|\right)$ can be calculated by (1). Normally, it is difficult to obtain the analytic solution of (1). Therefore, we can only obtain a numerical solution. After simulation operation, LINGO $^{\circledR}$ software is used to obtain the numerical solution. It has high speed, high accuracy, and lower pseudo value. Therefore, the solving process is realized by LINGO 8.0 software.

\subsection{Eliminating the Effect of Geomagnetic Field Variations and} Uneven Spatial Distribution. The geomagnetic field changes along with time and it has an uneven distribution in space [15]. Since the magnetic anomaly generated by the remote target is usually small, the geomagnetic field variation and uneven spatial distributions are important factors for affecting localization accuracy. Therefore, it is necessary to eliminate the influence of these factors when locating the magnetic target. We assume that the target does not reach the detection range at $t_{0}$ time. When the target is detected, the measured value $T_{i}$ of the $i$ th sensor at $t$ time consists of three parts, which can be expressed as follows:

$$
\begin{aligned}
T_{i}\left(t, x_{i}, y_{i}, z_{i}\right)= & T_{0}\left(t_{0}, x_{s i}, y_{s i}, z_{s i}\right)+\Delta T\left(t-t_{0}\right) \\
& +\Delta T_{\text {target }}\left(t, x_{i}, y_{i}, z_{i}\right),
\end{aligned}
$$

where $\left(x_{s i}, y_{s i}, z_{s i}\right)$ denotes the position of the $i$ th sensor. $\left(x_{i}, y_{i}, z_{i}\right)$ denotes the position of the target relative to the position of the $i$ th sensor. $T_{0}\left(t_{0}, x_{s}, y_{s}, z_{s}\right)$ is the geomagnetic field measured by the $i$ th sensor at $t_{0}$ time. $\Delta T\left(t-t_{0}\right)$ is the value of the geomagnetic field variations in the time period $t-$ $t_{0} . \Delta T_{\text {target }}\left(t, x_{i}, y_{i}, z_{i}\right)$ is the magnetic anomaly measured by the $i$ th sensor at $t$ time. Because the geomagnetic field varies slowly in a large-scale space, the change of the geomagnetic field with time is basically synchronous in the local space. Similarly, the measured value $T_{j}$ of the $j$ th sensor at $t$ time can be expressed as

$$
\begin{aligned}
T_{j}\left(t, x_{j}, y_{j}, z_{j}\right)= & T_{0}\left(t_{0}, x_{s j}, y_{s j}, z_{s j}\right)+\Delta T\left(t-t_{0}\right) \\
& +\Delta T_{\text {target }}\left(t, x_{j}, y_{j}, z_{j}\right) .
\end{aligned}
$$

The difference of magnetic anomaly $\Delta T$ measured by the two sensors can be expressed as

$$
\begin{aligned}
\Delta T= & \Delta T_{\text {target }}\left(t, x_{i}, y_{i}, z_{i}\right)-\Delta T_{\text {target }}\left(t, x_{j}, y_{j}, z_{j}\right) \\
= & T_{i}\left(t, x_{i}, y_{i}, z_{i}\right)-T_{0}\left(t_{0}, x_{s i}, y_{s i}, z_{s i}\right) \\
& -\left[T_{j}\left(t, x_{j}, y_{j}, z_{j}\right)-T_{0}\left(t_{0}, x_{s j}, y_{s j}, z_{s j}\right)\right] \\
= & {\left[T_{i}\left(t, x_{i}, y_{i}, z_{i}\right)-T_{j}\left(t, x_{j}, y_{j}, z_{j}\right)\right] } \\
& -\left[T_{0}\left(t_{0}, x_{s i}, y_{s i}, z_{s i}\right)-T_{0}\left(t_{0}, x_{s j}, y_{s j}, z_{s j}\right)\right] .
\end{aligned}
$$

It can be seen from (4) that $\Delta T$ is not affected by time. And it can also eliminate the influence of the uneven distribution of the geomagnetic field. Therefore, $\Delta T$ is used in the calculation for target localization.

\section{Localization Experiment and Data Analysis}

3.1. Experimental Setup. In order to test the correctness of (1) and verify the effectiveness of the proposed method, we carried out the experiments on the localization of a moving target in the suburbs of Qiqihar city, China. In the experiment, we had only two optical pumped magnetometers (CS-L). This magnetometer had high sensitivity, and intrinsic noise was about $0.6 \mathrm{pT} / \sqrt{ } \mathrm{Hz}$ at $1 \mathrm{~Hz}$. We constructed a onedimensional sensor array shown in Figure 3 to estimate $y$ 


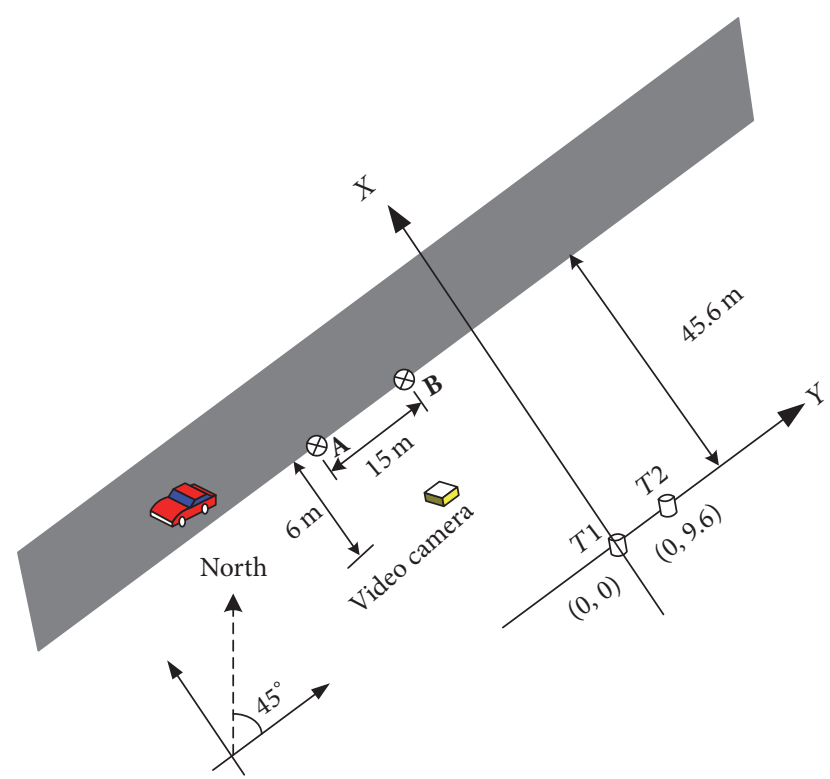

FIGURE 3: Schematic diagram of the experiment.

positions of the moving target using the proposed method. Two magnetometers were located on the $y$-axis and were spaced apart by $9.6 \mathrm{~m}$. The road was parallel to the $y$-axis with $x=45.6 \mathrm{~m}$. The angle between the $y$-axis and the north was 45 degrees. In addition, the local geomagnetic inclination was 64 degrees and the geomagnetic declination was -9 degrees. The car, as the target with constant velocity, was driven on the road. And we made two marked points on the side of the road; the distance between them was $15 \mathrm{~m}$. Then, we used a video camera to record the time at which the car passed the marks. According to the recorded image, we calculated the $y$ positions of the car and considered them as the true values. Meanwhile, we can also estimate the magnitude of the magnetic moment using the information of the marker positions.

3.2. Total Geomagnetic Field Measurement. Two optical pumped magnetometers were set to record the geomagnetic field at the same time. And the data were acquired at a sampling rate of $2 \mathrm{~Hz}$. One part of the total magnetic field recorded by the magnetometers is shown in Figure 4.

In Figure 4, the horizontal axis represents time and the vertical axis represents the values of the magnetic field. The blue curve represents the geomagnetic field data measured by magnetometer 1 . It can be seen from the blue curve that the total magnetic field varies along with time. Due to the geomagnetic field variations, it was difficult for us to determine whether there was a car through. And it is impossible to directly locate the position of the target using the measured data with the geomagnetic field variations. Since the change of the geomagnetic field with time is basically synchronous in the local space, we can eliminate the geomagnetic field variations according to (4). And the difference of the geomagnetic field between sensor 1 and sensor 2 is shown as a red curve. It can be easily seen that the geomagnetic field variations are eliminated. And the red line

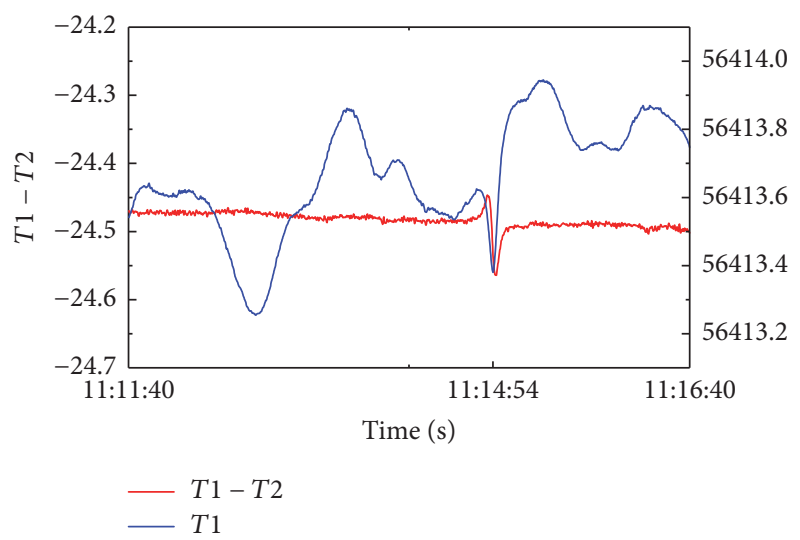

FIgURE 4: Total magnetic field measured by the magnetometers.

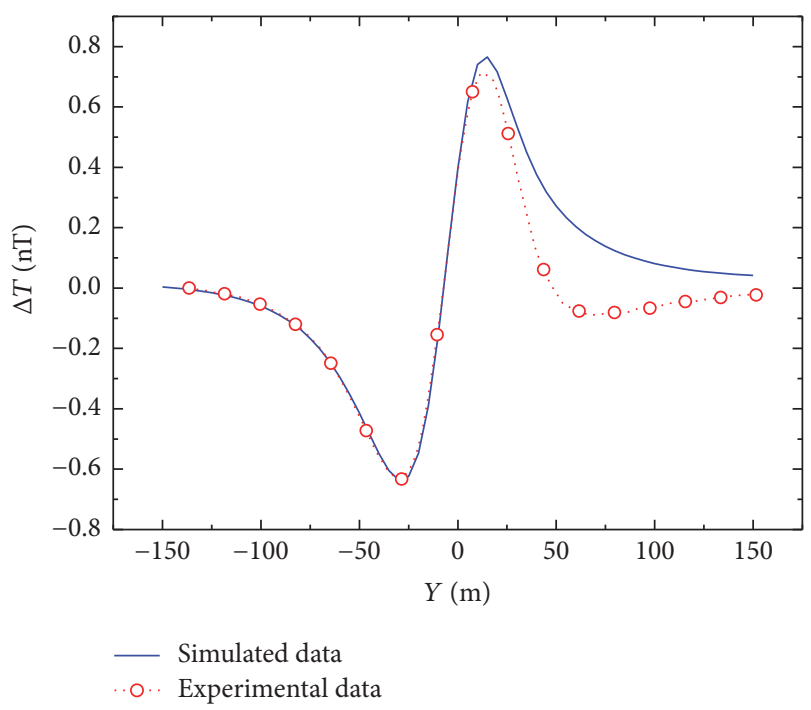

FIGURE 5: $\Delta T$ generated by the truck.

has basically become a straight line without the target. When the car passed the array at 11:14:54, the change of the magnetic anomaly can be easily determined using the difference of the geomagnetic field measured by magnetometers.

\subsection{Analysis of the Geomagnetic Total Field Localization} Experiment Data. A small truck with a mass of 10 tons passed through the marked points in $1.67 \mathrm{~s}$. We calculated the velocity of the target at $32.4 \mathrm{~km} / \mathrm{h}$. And we estimated the magnetic moment of $5513 \mathrm{~A} / \mathrm{m}^{2}$ according to the magnetic anomaly measured by the magnetometers at the time when the truck passed the marked points. Then, we assumed that the magnetic moment of the truck remained constant during the moving process. According to the positions of the marked points and the velocity, the $y$ positions of the target were calculated at each moment. Then, the magnetic anomalies generated by the target were simulated according to formula (4). The simulation is shown in Figure 5. It can be seen that the simulated magnetic anomalies were close to the 


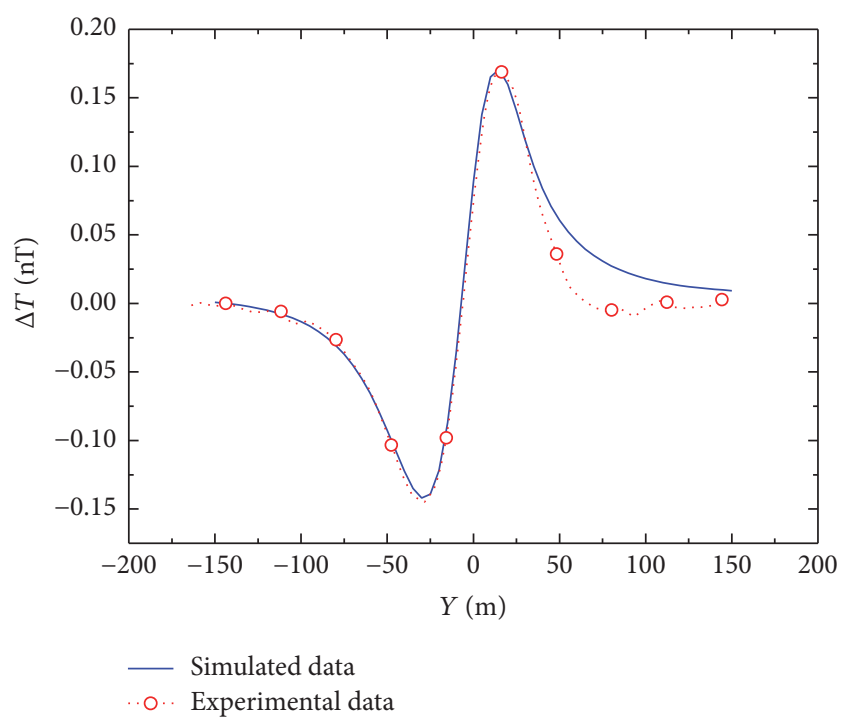

FIgURE 6: $\Delta T$ generated by the SUV.

experimental data when $y<22.3 \mathrm{~m}$. The experimental data can be well described by formula (4). When $y>22.3 \mathrm{~m}$, the error between the simulated data and the experimental data became larger.

Then, a small SUV passed through the marked points in $0.94 \mathrm{~s}$. We calculated the velocity of the target at $57.6 \mathrm{~km} / \mathrm{h}$. And we estimated the magnetic moment of $1230 \mathrm{~A} / \mathrm{m}^{2}$ according to the magnetic anomaly measured by the magnetometers at the time when the SUV passed the marked points. Then, we assumed that the magnetic moment remained constant during the moving process. According to the positions of the marked points and the velocity, the $y$ positions of the target were calculated at each moment. Then, the magnetic anomalies generated by the target were simulated according to formula (4), shown in Figure 6. It can be seen that the simulated magnetic anomalies were close to the experimental data when $y<27.6 \mathrm{~m}$. And the experimental data can be well described by formula (4). When $y>27.6 \mathrm{~m}$, the deviation between the theoretical data and the experimental data became larger.

3.4. Localization Results. In the experiments, when the magnetic fields were measured by magnetometers, the difference of the magnetic anomaly $\Delta T$ can be easily obtained. Since the parameters $\left(x, z, D, \theta, \varphi,\left|\mathbf{P}_{m}\right|\right)$ were known, the $y$ position of the target can be calculated according to formula (4). However, formula (4) is a high order nonlinear equation. We can only obtain the numerical solution. Therefore, we used LINGO software to solve the nonlinear equation.

The $y$ positions of the small truck calculated by the proposed method are shown in Figure 7. It can be seen that the calculated positions of the target were close to the true positions when $y<22.3 \mathrm{~m}$. When $y>22.3 \mathrm{~m}$, the error of the calculated positions and the true values became larger. This is because the magnetic anomalies measured by the magnetometers cannot be well described by formula (4). The

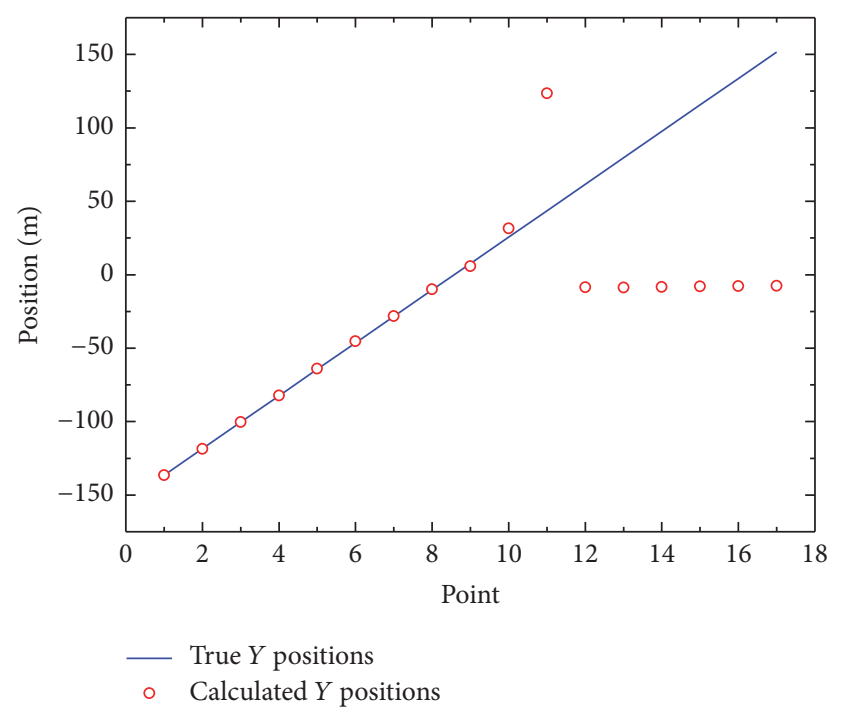

FIgURE 7: Localization results of a small truck.

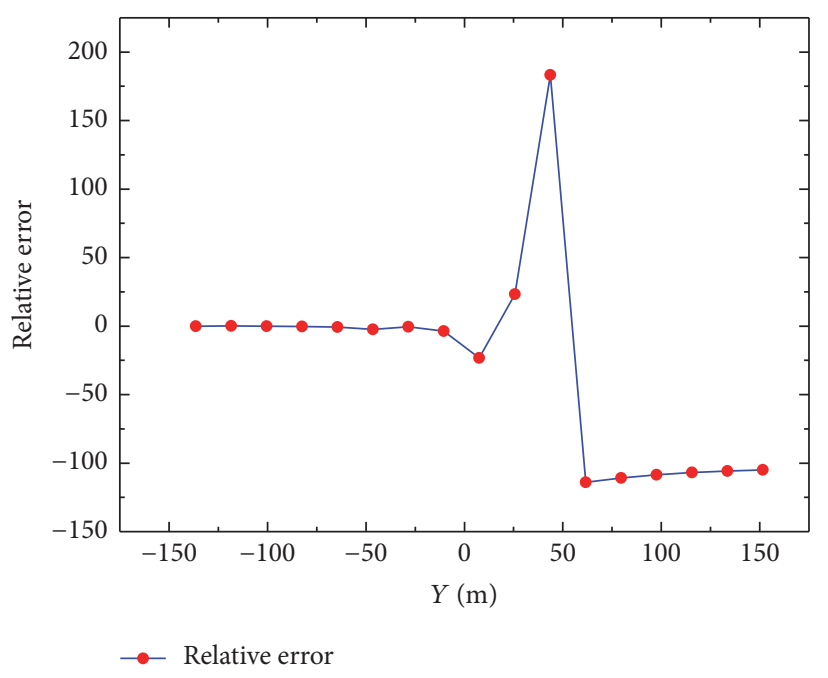

FIGURE 8: Relative error of localization of a small truck target.

relative error of the truck localization is shown in Figure 8. When the positions of the target were located at the range $y<$ $0 \mathrm{~m}$, the relative error of localization was less than $4 \%$. When the positions of the target were located at the range $0<y<$ $22.3 \mathrm{~m}$, the relative error of localization was relatively large. However, the error of localization is less than $4 \mathrm{~m}$, which is less than the length of the truck. When the positions of the target were located at the range $y>22.3 \mathrm{~m}$, the relative error of localization was very large.

The $y$ positions of the small SUV calculated by the proposed method are shown in Figure 9. Similar to localization of the truck, the calculated positions of the target were close to the true positions when $y<27.6 \mathrm{~m}$. When $y>27.6 \mathrm{~m}$, the error of the calculated positions and the true values also became larger. The same reason was that the magnetic anomalies measured by the magnetometers cannot be well 


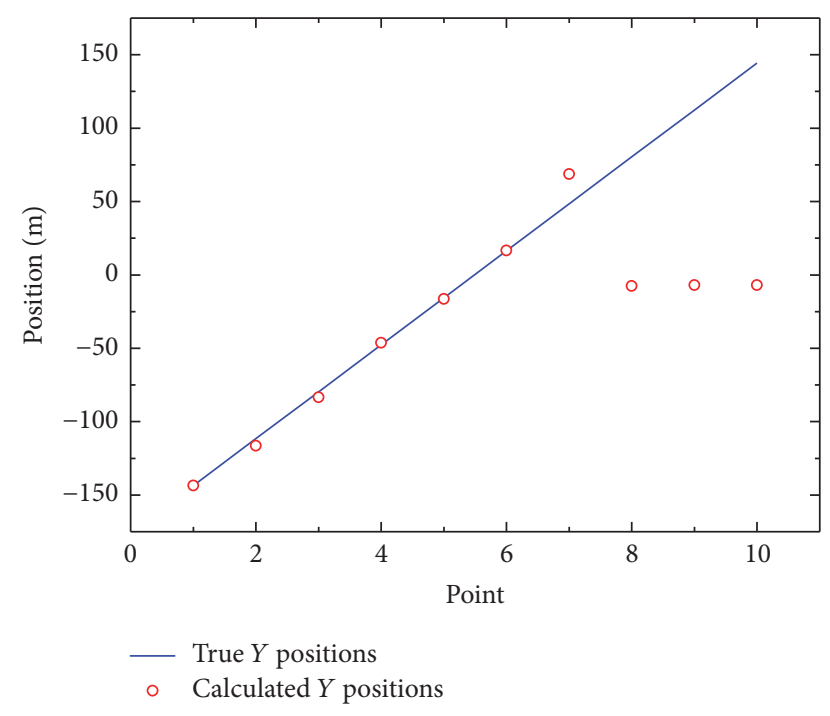

FIgURE 9: Localization results of a small SUV.

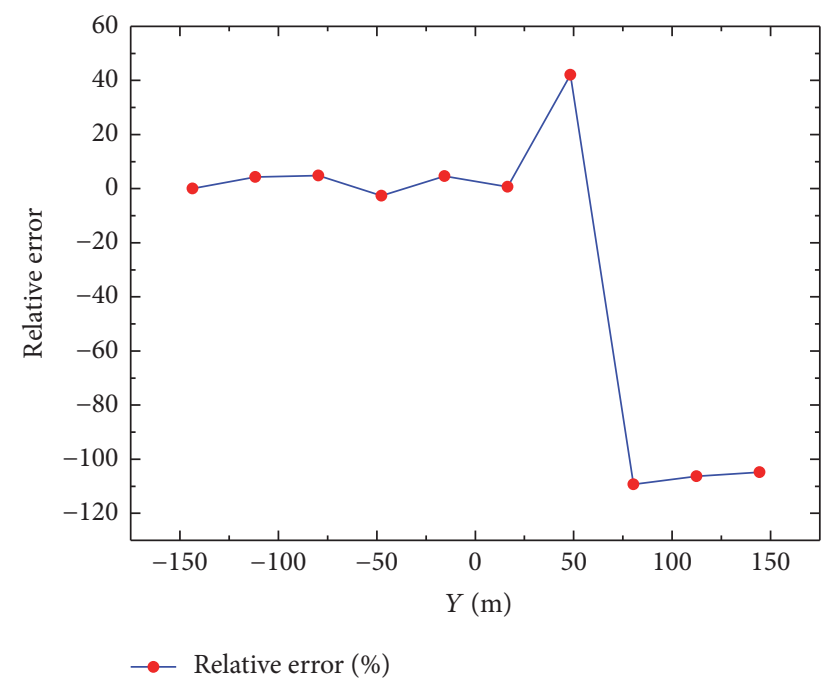

FIGURE 10: Relative error of localization of a small SUV.

described by formula (4). The relative error of the SUV localization is shown in Figure 10. When the positions of the target were located at the range $y<27.6 \mathrm{~m}$, the relative error of localization was less than $5 \%$. When the position of the target was located at the range $y>27.6 \mathrm{~m}$, the relative error of localization was very large.

\section{Discussions}

In the experiment of the truck, when $y>22.3 \mathrm{~m}$, the magnetic anomalies $\Delta T$, which are simulated according to the known information and formula (4), are not well consistent with the measured data. And in the experiment of the SUV, when $y>27.6 \mathrm{~m}$, the magnetic anomalies $\Delta T$ are also not well consistent with the measured data. This leads to a relatively large error of the target's localization. We have reviewed the

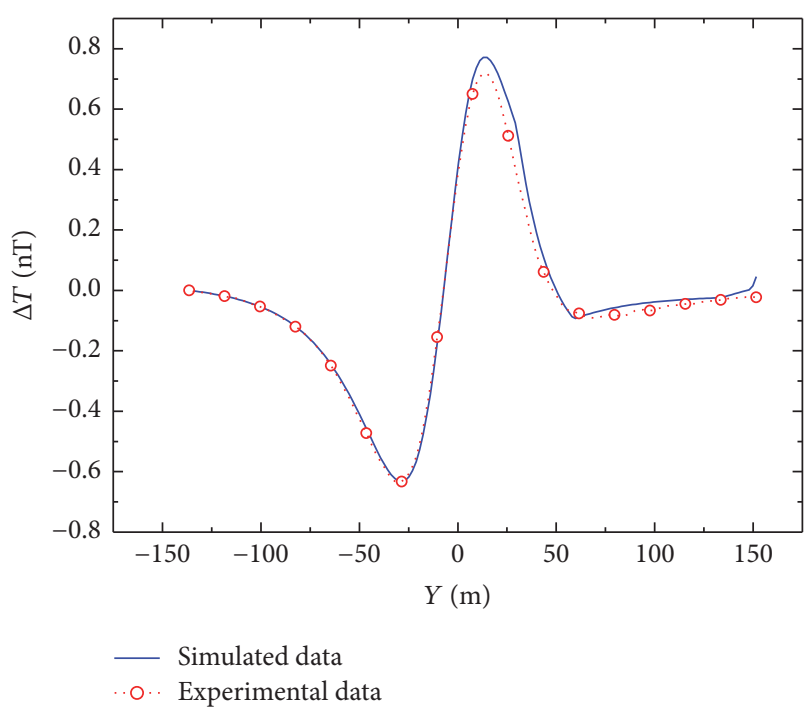

FIGURE 11: $\Delta T$ generated by the truck considering the ferromagnetic materials on the roadside.

experimental records. And we found that there are ferromagnetic materials (iron fences) on the roadside from $y>$ $30 \mathrm{~m}$. These ferromagnetic materials can generate magnetic fields. And they are superimposed on the geomagnetic field, which results in a change in the magnetic field near the ferromagnetic material. In [13], the soft magnetic moment $\mathbf{P}_{m}^{\mathbf{s}}$ of the target is parallel to the ambient magnetic field $\mathbf{T}$, and the magnitude can be expressed as

$$
\left|\mathbf{P}_{\mathbf{m}}^{\mathbf{s}}\right|=\frac{D}{\mu_{0}}|\mathbf{T}|,
$$

where $D$ is a target characteristic scalar constant. It can be seen that the magnitude of the soft magnetic moment is proportional to the magnitude of the ambient magnetic field.

Due to the presence of the ferromagnetic materials on the roadside when $y>30 \mathrm{~m}$, the ambient magnetic field should be considered as a function about $y$ as $|\mathbf{T}|=f(y)$. According to (5), the magnetic moment of the target should be considered as a function about $y$ when the $y$ positions' target is greater than a certain value. However, we considered that the magnetic moment remained constant in the calculation. This is the main reason why there is a relatively large error between the simulated magnetic anomalies and the measured data when $y$ is greater than a certain value.

We built a model to describe the ferromagnetic materials which are located on the roadside. The ferromagnetic materials consist of a series of magnetic dipoles. The distance between the adjacent magnetic dipoles is $20 \mathrm{~cm}$ and the distribution of the magnetic dipoles is set as $x=46 \mathrm{~m}, 30 \mathrm{~m}$ $\leq y \leq 150 \mathrm{~m}$, and $z=0 \mathrm{~m}$.

We consider this model in the data process. The magnetic anomalies generated by the small truck are resimulated and the result is shown in Figure 11. And the magnetic anomalies generated by the small SUV are resimulated and the result is shown in Figure 12. It can be seen that the simulated data 


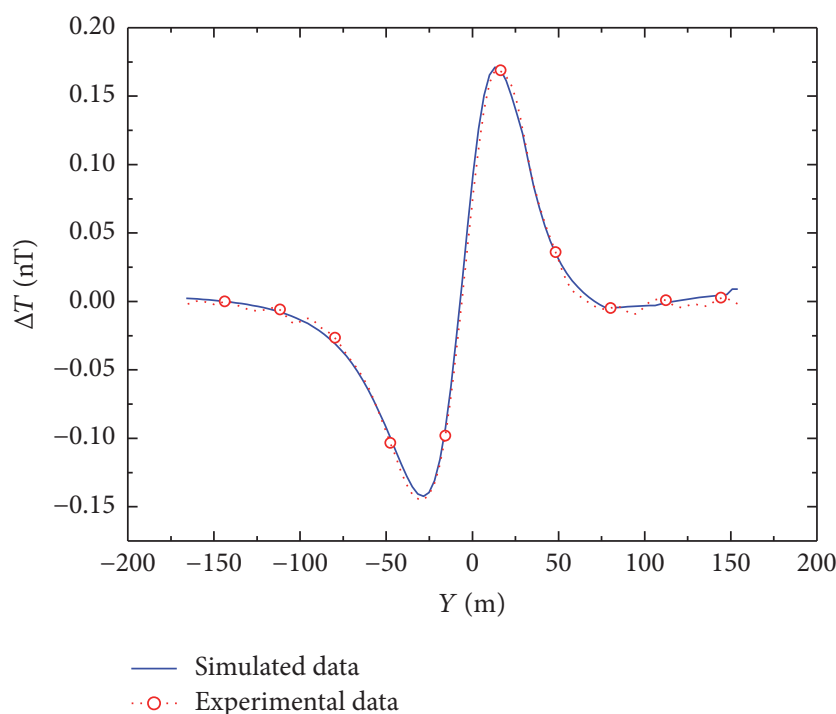

FIGURE 12: $\Delta T$ generated by a small SUV considering the ferromagnetic materials on the roadside.

are close to the experimental data in the whole range of the detection after considering this model. This indicates that the deviations between the simulated data and the experimental data in Figures 5 and 6 are caused by the ferromagnetic materials on the roadside.

\section{Conclusion}

In this paper, we present an array with the total field magnetometers and propose a method for the localization of the target based on the total geomagnetic field. In the proposed method, the magnetic target is considered as the magnetic dipole and the nonlinear equations for the target localization are obtained. In order to eliminate the effect of geomagnetic field variations and uneven spatial distribution, we use the difference of the geomagnetic field measured by the two magnetometers in the calculation. The nonlinear equations for localization algorithm are solved by LINGO software. The experiments were carried out in the suburbs of the city. We constructed an array with two optical pumped magnetometers and used the proposed method to locate the $y$ positions of the car which was driven through the array. The results show that the estimated $y$ positions of the target were close to the true values. And the relative error of the localization is less than $5 \%$ by using the total geomagnetic field to locate the target.

\section{Conflicts of Interest}

The authors declare that they have no conflicts of interest.

\section{References}

[1] W. Yang, C. Hu, M. Li, M. Q.-H. Meng, and S. Song, "A new tracking system for three magnetic objectives," IEEE Transactions on Magnetics, vol. 46, no. 12, pp. 4023-4029, 2010.

[2] W. M. Wynn, "Detection, localization, and characterization of static magnetic dipole sources," in Detection and Identification of Visually Obscured Targets, pp. 337-374, 1999.

[3] T. Nara, S. Suzuki, and S. Ando, "A closed-form formula for magnetic dipole localization by measurement of its magnetic field and spatial gradients," IEEE Transactions on Magnetics, vol. 42, no. 10, pp. 3291-3293, 2006.

[4] W. M. Wynn, "Magnetic dipole localization using the gradient rate tensor measured by a five-axis magnetic gradiometer with known velocity," in Proceedings of the SPIE's 1995 Symposium on OE/Aerospace Sensing and Dual Use Photonics, pp. 357-367, Orlando, FL, USA, 1995.

[5] A. Sheinker, B. Ginzburg, N. Salomonski, P. A. Dickstein, L. Frumkis, and B.-Z. Kaplan, "Magnetic anomaly detection using high-order crossing method," IEEE Transactions on Geoscience and Remote Sensing, vol. 50, no. 4, pp. 1095-1103, 2012.

[6] G. Iacca, F. L. Bakker, and H. Wörtche, "Real-time magnetic dipole detection with single particle optimization," Applied Soft Computing Journal, vol. 23, pp. 460-473, 2014.

[7] Y. I. N. Gang, Z. Yingtang, L. Zhining, F. Hongbo, and R. Guoquan, "Detection of ferromagnetic target based on mobile magnetic gradient tensor system," Journal of Magnetism and Magnetic Materials, vol. 402, pp. 1-7, 2016.

[8] U. Marschner and W.-J. Fischer, "Indirect measurement of a bar magnet position using a hall sensor array," IEEE Transactions on Magnetics, vol. 43, no. 6, pp. 2728-2730, 2007.

[9] R. Liu and H. Wang, "Detection and localization of improvised explosive devices based on 3-axis magnetic sensor array system," Procedia Engineering, vol. 7, pp. 1-9, 2010.

[10] Y. Sui, G. Li, S. Wang, and J. Lin, "Asphericity errors correction of magnetic gradient tensor invariants method for magnetic dipole localization," IEEE Transactions on Magnetics, vol. 48, no. 12, pp. 4701-4706, 2012.

[11] J. Lenz and A. S. Edelstein, "Magnetic sensors and their applications," IEEE Sensors Journal, vol. 6, no. 3, pp. 631-649, 2006.

[12] P. Stavrev and D. Gerovska, "Magnetic field transforms with low sensitivity to the direction of source magnetization and high centricity," Geophysical Prospecting, vol. 48, no. 2, pp. 317-340, 2000.

[13] N. Wahlström and F. Gustafsson, "Magnetometer modeling and validation for tracking metallic targets," IEEE Transactions on Signal Processing, vol. 62, no. 3, pp. 545-556, 2014.

[14] L. Fan, C. Kang, X. Zhang, and S. Wan, "Real-Time Tracking Method for a Magnetic Target Using Total Geomagnetic Field Intensity," Pure and Applied Geophysics, vol. 173, no. 6, pp. 20652071, 2016.

[15] J. E. McFee and Y. Das, "Determination of the Parameters of a Dipole by Measurement of its Magnetic Field," IEEE Transactions on Antennas and Propagation, vol. 29, no. 2, pp. 282-287, 1981.

\section{Acknowledgments}

This work was supported by the National Natural Science Foundation of China (no. 61174192). 


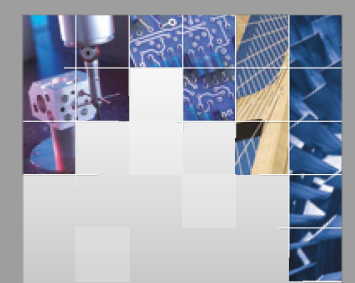

\section{Enfincering}
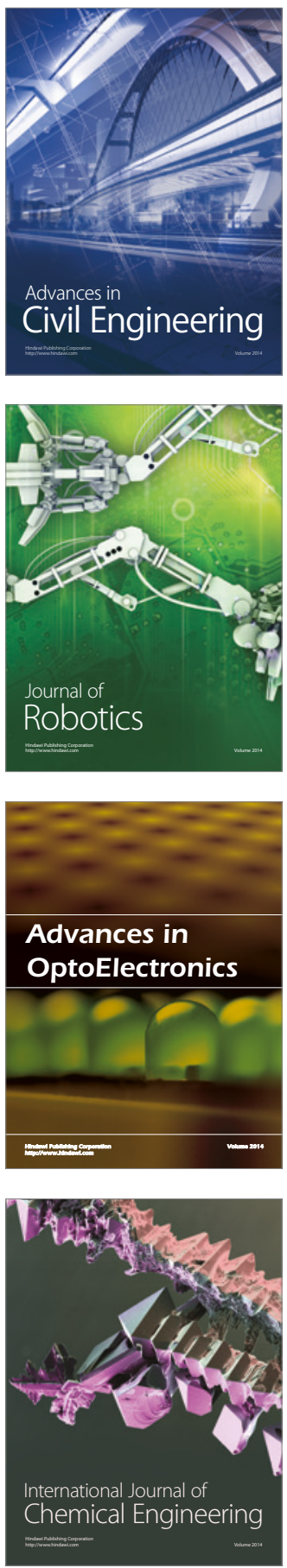

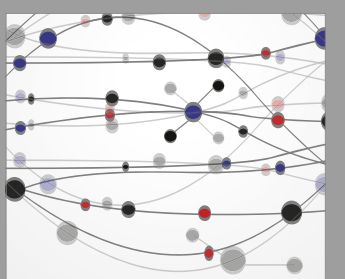

The Scientific World Journal

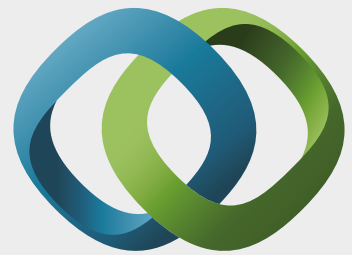

\section{Hindawi}

Submit your manuscripts at

https://www.hindawi.com
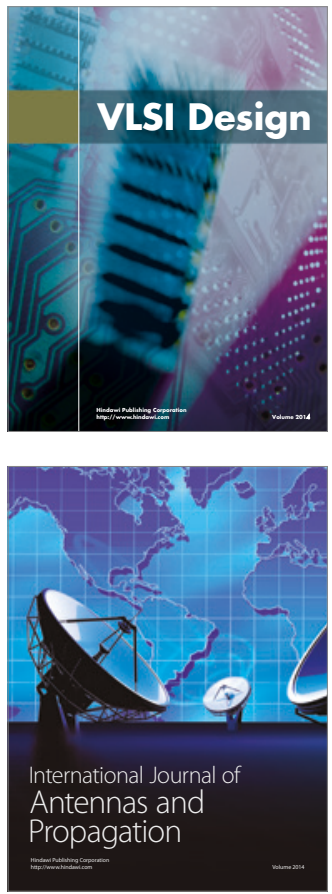

\section{Rotating}

Machinery
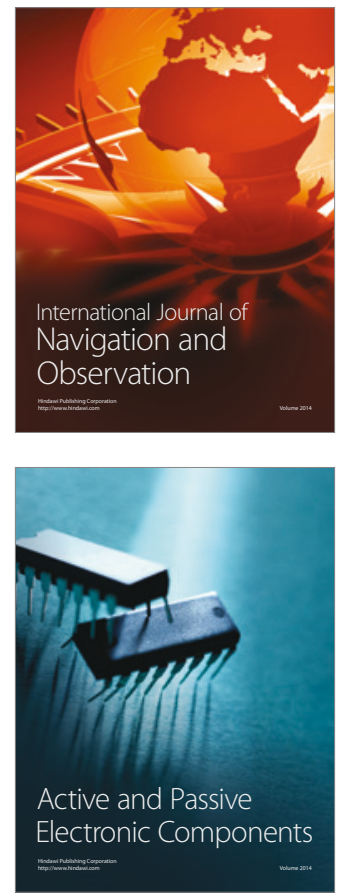
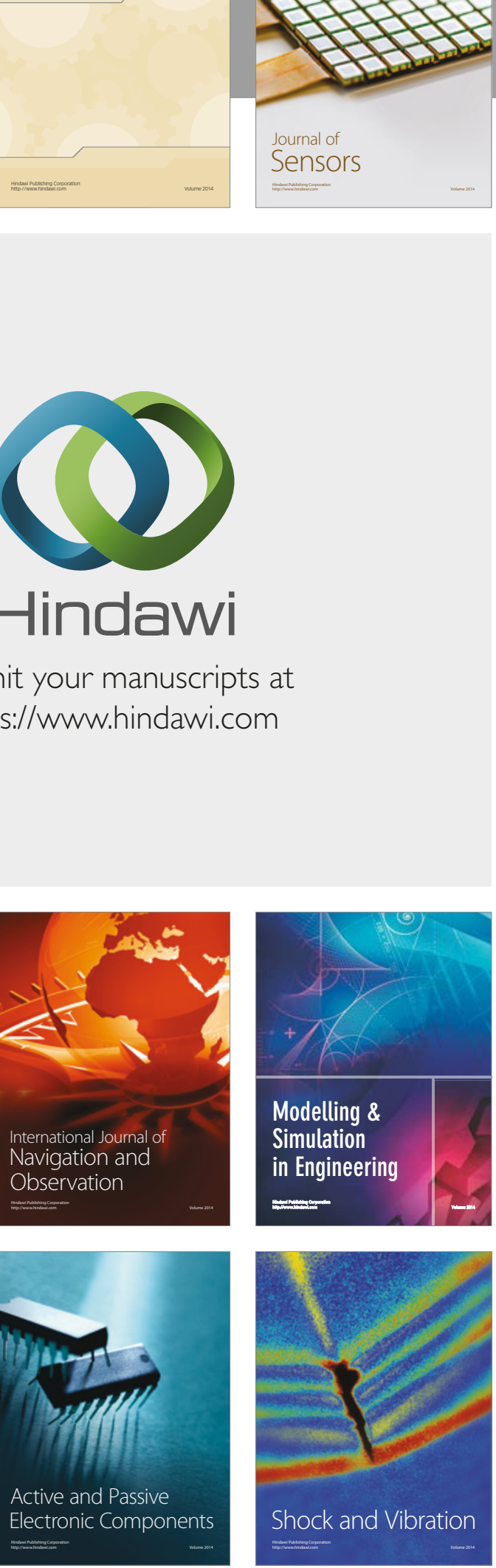
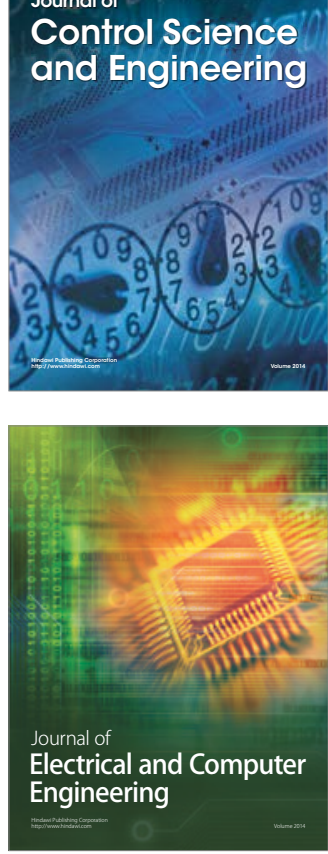

Distributed

Journal of

Control Science

and Engineering
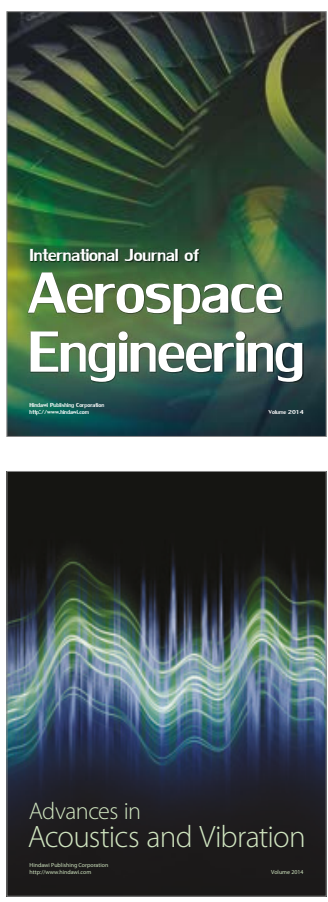

Sensor Networks 